\title{
Ultralow thermal sensitivity of phase and propagation delay in hollow-core fibers
}

\author{
Eric Numkam Fokoua, Marco N. Petrovich, Tom Bradley, Francesco Poletti, David J. \\ Richardson, and Radan Slavík* \\ * Optoelectronics Research Centre, University of Southampton, SO17 1BJ, United Kingdom \\ ernf1g10@orc.soton.ac.uk
}

\begin{abstract}
We discuss our recent work on the thermal sensitivity of phase and propagation delay in hollow-core photonic bandgap fibers, showing in the case of the latter that it can be reduced to zero through careful design of the fiber structure.
\end{abstract}

\section{INTRODUCTION}

In optical fiber communications, the primary application sphere for optical fibers, data-carrying optical signals are usually considered immune to thermal fluctuations across data links. However, changes in environmental variables such as temperature or humidity all induce changes in the properties of the materials making the optical fiber. While these changes do not generally impact the power of propagating optical signals or their relative phase differences (detected in communication systems), they affect both the propagation time and the total phase accumulated by such signals [1]. Thermally-induced changes in propagation time and phase have far-reaching implications and are highly undesirable in a host of applications for which optical fibers are uniquely suited. These include interferometric sensors such as gyroscopes in which the phase thermal sensitivity ultimately results in an additional error from the Shupe effect, and applications requiring very stable time delays such as in precise time and frequency transfer for example [2-3]. Furthermore, a number of emerging applications including future $5 \mathrm{G}$ networks will benefit tremendously from the use of optical fibers in which both phase and delay are immune to environmental changes.

In a standard single mode fiber, the propagation delay changes with temperature at a rate of nearly $40 \mathrm{ps} / \mathrm{km} / \mathrm{K}$ and its phase sensitivity is approximately $8 \mathrm{ppm} / K$ [1-2]. The main route to reducing this thermal sensitivity has been through the application of specialty liquid crystal coatings with negative coefficient of thermal expansion (CTE) to counteract thermally induced fibre elongation and refractive index changes [1]. Such specially coated low sensitivity fibers are now commercially available, with the very best achieving a delay sensitivity of $3.7 \mathrm{ps} / \mathrm{km} / \mathrm{K}$, more than a tenfold reduction relative to standard SMF-28 fibres. However, many applications are set to benefit greatly from further reduction in temperature sensitivity beyond these levels. Since changes in the glass refractive index with temperature account for over $95 \%$ of the delay thermal sensitivity in standard fibers, it follows that by making light propagate in air, such as in a hollowcore fiber (HCF), the fiber's thermal sensitivity can be greatly reduced.

Here, we review our recent results where we have shown that hollow-core photonic bandgap fibres (HCPBGFs) offer significantly reduced thermal sensitivity than solid-core fibres in terms of - propagation time/phase. Indeed, we have shown for the first time both theoretically and experimentally, that without recourse to specialty coatings, it is possible to reduce the thermal sensitivity of the propagation delay to zero in these fibers by designing them so that any thermal elongation is compensated by an increase in the mode group velocity[4-5].

\section{THERMAL SENSITIVITY IN HC-PBGFS}

\section{A. Theory}

The potential of HCFs for low temperature sensitivity applications is understood through the following analysis. The signal propagation delay $\tau$, and the phase $\phi$ are defined by:

$$
\begin{aligned}
\tau & =\left(n_{g} L\right) / c \\
\phi & =\beta L,
\end{aligned}
$$

where $L$ is the fibre length, $\beta$ the mode propagation constant, $n_{g}=c \partial \beta / \partial \omega$ the group index of the mode and $c$ the speed of light in vacuum. The thermal sensitivity of these two quantities are quantified through the thermal coefficient of delay (TCD) and the phase thermal constant defined (S) as:

$$
\begin{aligned}
T C D & =\frac{1}{L} \frac{d \tau}{d T}=\frac{1}{c}\left(\frac{n_{g}}{L} \frac{d L}{d T}+\frac{d n_{g}}{d T}\right) \\
S & =\frac{1}{\phi} \frac{d \phi}{d T}=\frac{1}{L} \frac{d L}{d T}+\frac{1}{\beta} \frac{d \beta}{d T}
\end{aligned}
$$

The first term in both Eqs. (3) and (4) relates to the thermal elongation of the fiber. The second term in both equations originates from thermally-induced changes in the mode propagation constant. The propagation constant changes with temperature through three effects: (i) the transverse thermal expansion of the fiber, (ii) the photoelastic effect which changes the glass refractive index due to the thermal stresses and finally, (iii) the thermo-optic effect which further alters the glass index. 
In [5], we determine the TCD and by extension, the phase thermal constant, through a rigorous perturbation treatment. However, for HC-PBGFs, profound physical insight can be gained through very simple qualitative considerations. Indeed, to the zeroth order approximation, the processes outlined above all contribute to a red-shift in the position of the photonic bandgap of $\sim \Delta \lambda / \Delta T=$ $23.2 \mathrm{pm} / \mathrm{K}$. Assuming that all important optical properties of the fiber are simply red-shifted along with the bandgap, a quick estimate gives:

$$
\begin{aligned}
& T C D=\varepsilon_{z z} \frac{n_{g}}{c}-D \frac{\Delta \lambda}{\Delta T} \\
& S=\varepsilon_{z z}+\frac{1}{\lambda}\left(\frac{n_{g}}{n_{e f f}}-1\right) \frac{\Delta \lambda}{\Delta T}
\end{aligned}
$$

where $\varepsilon_{z z}$ is the effective thermal elongation coefficient, $D$ the chromatic dispersion in $s^{2} / m$ and $n_{e f f}$ the mode's effective index of propagation. Eq.(5) shows that for a large core HCF in which dispersion is low, the TCD is dominated by the elongation term. With typically soft polymer coatings, $\varepsilon_{z z}$ reduces to the CTE of silica and with the typical value of $n_{g} \sim 1.003$, it follows that $T C D \approx 1.8 \mathrm{ps} / \mathrm{km} / K$, nearly 20 times lower than in the SMF [4]. Because both $n_{\text {eff }}$ and $n_{g}$ are very close to 1 , Eq.(6) says that $S$ too is very close to the CTE of silica, or $0.55 \mathrm{ppm} / \mathrm{K}$, also about 15 times lower than in the SMF. Crucially, Eq.(5) reveals that further TCD reduction is possible, even to zero or made negative, simply by engineering the fiber dispersion. Indeed, it predicts that the TCD vanishes when $D=79 \mathrm{ps} / \mathrm{nm} \cdot \mathrm{km}$.

In proof-of-principle experiments, we have shown first an 18 times improvement of the TCD in a large core HCPBGF as compared to SMF-28, that is, about two times improvement over the best-performing specialty-coated solid-core fibres, and later demonstrated for the first time that the TCD can be reduced to zero in a fiber with a smaller core size. This makes HC-PBGFs the bestperforming fibres available today and the ultimate solution for a number of emerging temperature sensitive applications

\section{B. Experiments}

The setup sketched in Fig. 1(a) was used for proof-ofprinciple demonstrations. Few meters of in house fabricated HCPBGF samples coiled on a $10 \mathrm{~cm}$ diameter drum were placed along with a high resolution thermometer between two metallic plates and placed onto a hot plate with closed-loop temperature control and inserted in one arm of a free-space balanced MachZehnder interferometer. The interferogram was recorded using an optical spectrum analyzer (OSA). The spectral phase was extracted by locating positions of constructive and destructive interference, and the delay calculated as its first derivative with respect to optical frequency. Fig. 1(b) shows the delay measured at $29^{\circ} \mathrm{C}$ and Fig.(c) the differential delay for 47,65 , and $82^{\circ} \mathrm{C}$ when compared to that measured at $29^{\circ} \mathrm{C}$. Fig. 1(d) shows the measured TCD as a function of wavelength (black curve) along with simulated values (red curve). The agreement between both curves is remarkable and it can be appreciated that near
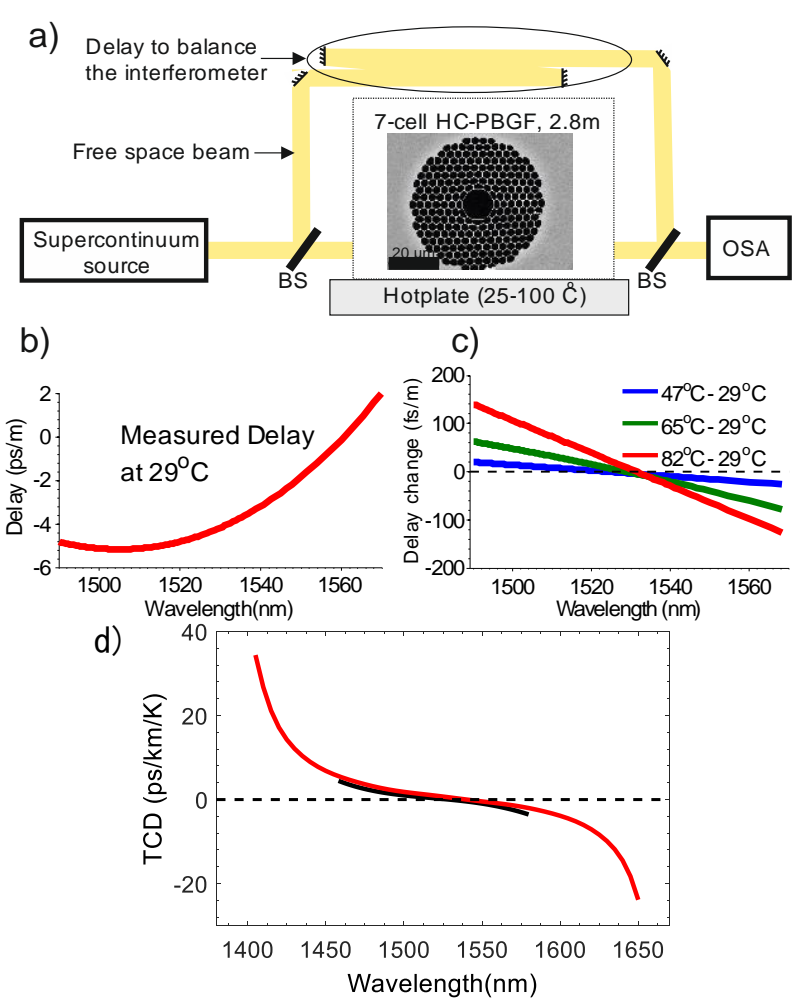

Figure 1: Experimental demonstration of ultralow thermal sensitivity in HC-PBGFs. a) Setup b) delay recorded at $29^{\circ} \mathrm{C}$, c) relative delay at 47,65 and $82^{\circ} \mathrm{C}$ and d) measured (black) and simulated (red) TCD

$1530 \mathrm{~nm}$, the TCD is exactly zero. Chromatic dispersion was measured in the fiber and we obtained the value of $83 \mathrm{ps} / \mathrm{hm} \cdot \mathrm{km}$ at $1530 \mathrm{~nm}$ [5], again in very good agreement with our simple analysis above.

\section{Conclusions}

We have demonstrated for the first time an optical fiber in which the propagation delay is fully insensitive to temperature variations $(T C D=0)$. This was achieved by compensating the thermally-induced fiber elongation with an increase in modal group velocity. The ability to provide such ultrastable latency will allow new approaches in well-established fields such as precise time and frequency transfer, time-synchronous data transfer in optical communications, and help open up emerging new fields such as $5 \mathrm{G}$ networks and relativistic geodesy.

\section{REFERENCES}

[1] H. Bousonville et al., "New phase stable optical fiber," Proceedings of BIW 2012, paper MOPG033, pp. 101-103, Newport News, VA, USA, 2012

[2] V. Dangui, H.K. Kim, M.J.F. Digonnet, and G.S. Kino, "Phase sensitivity of temperature of the fundamental mode in air-guiding photonic-bandgap fibers,". Opt. Express 13, 6669-6684 (2005).

[3] F. Loehl et al., "Measurement and stabilization of the bunch arrival time at FLASH,“ Proceedings of the EPAC 2008 conference, pp. 3360-3362, Genoa, Italy, 2008.

[4] R. Slavík et al, "Ultralow thermal sensitivity of phase and propagation delay in hollow core optical fibres," Sci. Rep. 5, 15447 (2015).

[5] E. Numkam Fokoua et al, "How to make the propagation time through an optical fiber fully insensitive to temperature variations," Optica 4(6) 659-668 (2017) 